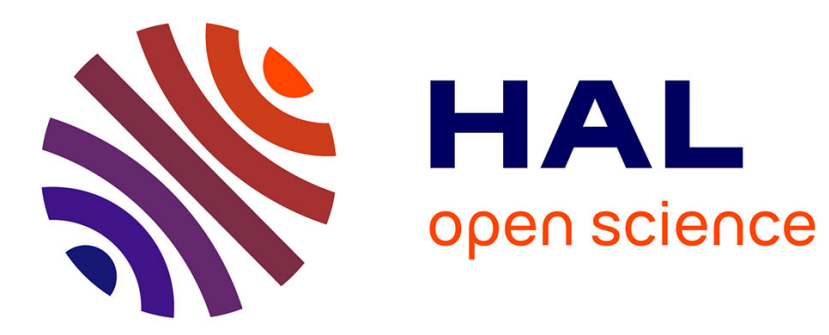

\title{
Role of an oscillatory electric field on the Lehmann rotation of cholesteric droplets \\ P. Oswald
}

\section{To cite this version:}

P. Oswald. Role of an oscillatory electric field on the Lehmann rotation of cholesteric droplets. European Physical Journal E: Soft matter and biological physics, 2020, 43 (2), 10.1140/epje/i202011935-2 . hal-02485306

\section{HAL Id: hal-02485306 https://hal.science/hal-02485306}

Submitted on 13 May 2020

HAL is a multi-disciplinary open access archive for the deposit and dissemination of scientific research documents, whether they are published or not. The documents may come from teaching and research institutions in France or abroad, or from public or private research centers.
L'archive ouverte pluridisciplinaire HAL, est destinée au dépôt et à la diffusion de documents scientifiques de niveau recherche, publiés ou non, émanant des établissements d'enseignement et de recherche français ou étrangers, des laboratoires publics ou privés. 


\title{
Role of an oscillatory electric field on the Lehmann rotation of cholesteric droplets
}

\author{
P. Oswald ${ }^{\mathrm{a}}$
}

Univ Lyon, ENS de Lyon, Univ Claude Bernard, CNRS, Laboratoire de Physique, F-69342 Lyon, France

Received:

Abstract. This paper deals with the Lehmann rotation of banded cholesteric droplets subjected to a temperature gradient when they coexist with their own isotropic liquid. I show that their angular rotation velocity increases -in absolute value- when they are subjected to an additional AC electric field in the conducting regime. This velocity increase is correlated with a prolate distortion of the droplets and the probable presence of electrohydrodynamical toroidal circulation flows inside and outside the droplets. I propose that the coupling between these flows and the director field is responsible for the increase of the angular velocity of the texture. The origin of these flows is discussed qualitatively in the framework of the leaky dielectric model by taking into account the generation of charges both in the bulk via a Carr-Helfrich mechanism (Tarasov, Krekhow and Kramer model) and at the surface of the droplet (Taylor-Melcher model).

PACS. 61.30.Jf Defects in liquid crystals - 61.72.Lk Linear defects: dislocations, disclinations - 81.40.Lm Deformation, plasticity, and creep

\section{Introduction}

A cholesteric phase is a chiral nematic phase spontaneously twisted in one space direction $[1,2]$. The Lehmann effect is the continuous rotation of cholesteric droplets subjected

a e-mail: patrick.oswald@ens-lyon.fr to a temperature gradient. Discovered in 1900 by Otto Lehmann [3], this effect was re-observed recently both when the droplets coexist with their own isotropic liquid [4-6] and when they are dispersed in a different liquid that partly dissolves the liquid crystal (LC) [7]. The first 
explanation of the Lehmann effect was given by Leslie in 1968 in terms of thermomechanical coupling [8]. Unfortunately, this explanation fails to explain the more basic experimental observations both in the coexistence region $[9,10]$ and in the emulsions (for a review see Ref. [11]). Other models were recently proposed such as the meltinggrowth model to explain the rotation in the coexistence region [12] and a pure hydrodynamical model to explain the rotation in the emulsions [13]. Despite these efforts, the Lehmann effect is not yet completely understood [11] and further experimental investigations are still desirable.

For this reason, I studied the role of an electric field on the Lehmann rotation when the latter is applied in addition of the temperature gradient. My motivation came from a recent observation by Auernhammer et al. about the deformation of nematic droplets in an $\mathrm{AC}$ electric field [14]. These authors showed that 'strong' flows can develop within the isotropic liquid around the droplets, leading to their deformation. These flows resemble very much the Marangoni flows observed around the droplets in the Lehmann experiment with emulsified cholesterics $[11,15]$. Because these flows are responsible for the droplet rotation in that case, one can expect that a similar effect exists in the coexistence region when an $\mathrm{AC}$ electric field is applied to the droplets. The goal of this paper is to check this prediction.

I mention that, surprisingly and to the best of my knowledge, no systematic experiments were performed so far with an AC electric field. On the other hand, several experiments were conducted with a DC field, first in In- dia $[16,17]$ and more recently in Russia $[18,19]$. The result of all these experiments was that a DC electric field can make the cholesteric droplets rotate provided it is large enough. The rotation under DC field was initially explained in terms of electromechanical coupling $[16,17]$ by analogy with the Leslie thermomechanical coupling [1] but it was shown later that this coupling is much too small to explain the observations [20]. Another explanation was proposed recently by Tarasov et al. [21-24] in the framework of an electrohydrodynamical (EHD) model. In this model, charges are generated in the bulk of the droplets due to the local divergences of the flexoelectric polarization. Under a DC electric field, the Coulomb force acting on these charges generates flows inside the droplets, making their texture rotate. This model predicts a linear dependence between the rotation velocity and the field, which is observed experimentally, and leads to rotation velocities that are compatible with experiments. By contrast, this model based on flexoelectricity cannot lead to a continuous rotation of the droplets under AC field because of its linear dependence on the field.

The plan of the paper is as follows. In Section II, I give the details about the experimental setup and the samples used in this study. The experimental results about the droplet rotation velocity are given in Section III. In this section, the role of frequency and amplitude of the electric field is analyzed as well as the role of the concentration of ionic impurities, droplet diameter, temperature gradient and sample thickness. These results are then discussed in Section IV in the framework of two models. The 
first one is due to Tarasov, Krehkov and Kramer and only considers the bulk charges generated by the Carr-Helfrich mechanism $[1,2]$ associated with the anisotropic conduction in the cholesteric phase. The second one is based on the Taylor-Melcher model [25-27] and only accounts for the surface charges. Conclusions are drawn at the end of this section and new experiments are proposed to test the relative importance of these two models.

\section{Experimental details}

The experimental setup used to impose the temperature gradient is described in Ref. [4]. In brief, the sample is sandwiched between two transparent ovens regulated in temperature within $0.01^{\circ} \mathrm{C}$. A Leica Laborlux 12 Pol polarizing microscope equipped with a Hamamatsu C474295 digital camera is used to visualize the droplets. The temperature gradient $G$ across the LC layer can be calculated from the temperature difference $\Delta T$ between the top and bottom ovens by using the formula $G(\mathrm{~K} / \mathrm{m}) \approx$ $1750 \Delta T[4]$.

The samples are prepared between two transparent ITO (Indium Tin Oxide) electrodes covered with a $\sim$ $100 \mathrm{~nm}$-thick polymercaptan layer. This layer imposes a sliding planar anchoring to the cholesteric phase [28-30] which almost completely dewets in the coexistence region in the presence of the isotropic liquid. This dewetting favors the formation of droplets that have the shape of slightly truncated spheres as revealed by confocal microscopy [6]. Two Nickel wires are used as a spacer to fix the sample thickness which is precisely measured with a
USB4000 Ocean Optics spectrometer. The LC used is the 8CB (4-Octyl-4-biphenylcarbonitrile from Frinton Laboratories, USA) doped with 1.5wt\% R811 (R-(+)-4-(2-methyl butyl)phenyl-4-hexyloxybenzoate from Merck, Germany). The cholesteric pitch at the solidus temperature is measured by using the Cano-wedge technique $[1,2]: P \approx 6 \mu \mathrm{m}$ (right-handed cholesteric phase). The ionic impurity TBAB (tetrabutylammonium bromide from Sigma-Aldrich) is used to dope the LC and increase its conductivity and its charge relaxation frequency. A LCR meter HP 4284A is used to measure the electric impedance of the samples from which the dielectric constants and the charge relaxation frequencies are deduced (Appendix A). Measurements are performed with 5 different samples. Only sample 4 is doped with 0.11 wt $\%$ TBAB. For each sample the thickness and the charge relaxation frequencies in the isotropic $\left(f_{r}^{I}\right)$ and cholesteric $\left(f_{r}^{\perp}, f_{r}^{\|}\right)$phases are given in Table 1 . All the samples are sealed with NOA $61 \mathrm{UV}$ glue. In spite of this precaution, the samples degrade in contact with the glue and the polymercaptan, their freezing range slowly increasing in time, mainly during the first two weeks. On the other hand, their charge relaxation frequencies do not change much (by less than a factor of 2), even after three months, meaning that the polymercaptan layer and the UV glue do not introduce large amounts of ionic impurities.

\section{Results}

In this paper, I only analyze the rotation of the banded droplets which are by far the most numerous. In these 
Table 1. Thickness of the 5 samples used and their charge relaxation frequencies measured one day after filling with the LC in the cholesteric and isotropic phases.

\begin{tabular}{lllll}
\hline Sample $^{\circ}$ & $d(\mu \mathrm{m})$ & $f_{r}^{\|}(\mathrm{Hz})$ & $f_{r}^{\perp}(\mathrm{Hz})$ & $f_{r}^{I}(\mathrm{~Hz})$ \\
\hline 1 & 21 & 1700 & 1300 & 2150 \\
2 & 21.5 & 1150 & 881 & 1650 \\
3 & 21.6 & 1280 & 1000 & 1590 \\
4 & 21.8 & 66700 & 49400 & 75600 \\
5 & 39.8 & 1330 & 1000 & 1647 \\
\hline
\end{tabular}

droplets the helical axis is perpendicular to the bands and to the temperature gradient (for a complete description of their texture, see [31]). These droplets form spontaneously in the region of coexistence with the isotropic liquid, when the temperature is close to $38-39^{\circ} \mathrm{C}$. Under the action of the temperature gradient, the droplet (more rigorously, the droplet texture) rotates at constant angular velocity $\omega$, clockwise $(\omega<0)$ when the temperature gradient points upwards $(\Delta T>0)$ and anticlockwise $(\omega>0)$ when the temperature gradient is reversed $(\Delta T<0)$, which is expected for a right-handed cholesteric LC $(P>0)$. Experimentally, $\omega$ depends on many parameters: the temperature gradient $G$ (or the difference temperature $\Delta T$ ), but also the droplet radius $R$ and the sample thickness $d$

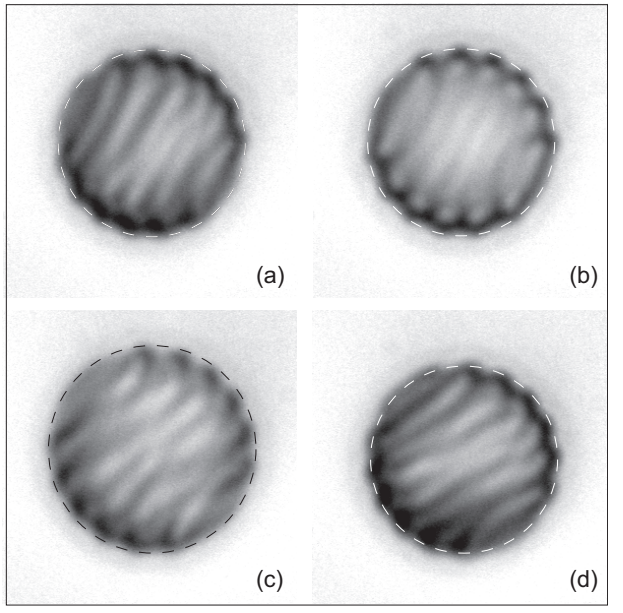

Fig. 1. Same banded droplet photographed in natural light when I successively impose $V=0$ (a), $V=4$ Vrms, $f=$ $10000 \mathrm{~Hz}$ (b) and $V=4$ Vrms, $f=100 \mathrm{~Hz}$ (c) and $V=0$ again (d). Note that in the meantime the droplet has rotated. The three white dashed circumferences have a radius of $9.9 \mu \mathrm{m}$ and the black dashed circumference has a radius of $10.7 \mu \mathrm{m}$. These photos show that the droplet radius increases when a low-frequency field is applied and does not change, within the experimental accuracy, when a high-frequency field is applied. Note in addition that the contrast and the periodicity of the bands change little when the field is applied, which indicates that the texture remains globally unchanged. The fact that the droplet recovers its initial radius when the field is switched off also indicates that the volume of the droplet does not change when the field is applied. Photos taken with sample 3.

(for a review, see [11]). In the present experiments, an AC electric field $E=\frac{\sqrt{2} V}{d} \cos (2 \pi f t)$ where $V$ is the applied voltage given in Vrms and $f$ the frequency, is also applied parallel to the temperature gradient. In this case, I show that the droplet rotation also depends on the electric field under certain conditions. I note now that in all of my ex- 
periments, the applied voltage is smaller than the critical voltage necessary to unwind the helix (around 6.5 Vrms in a $20 \mu \mathrm{m}$-thick sample and twice more in a $40 \mu \mathrm{m}$-thick sample) $[1,2]$. This is important to not change too much the internal texture of the droplets and the band periodicity inside. In the following, I analyze separately the effects of the different parameters, by starting with the electric field which is the focal point of my study.

\subsection{Role of the electric field}

The main observation of this paper is that the droplets flatten and rotate faster when they are subjected to a low-frequency AC electric field. By contrast, they remain unchanged and rotate at the same velocity as with no field when a high-frequency field is applied. In the following, this velocity is denoted by $\bar{\omega}$. The change of shape is evidenced in Fig. 1 showing the same droplet before and after the application of a low and high-frequency electric field. The change of velocity is shown in video S1. At the beginning of the movie, $V=0$ and the droplet rotates slowly clockwise under the sole action of the temperature gradient $\left(\Delta T=2^{\circ} \mathrm{C}\right)$. At time $\mathrm{t}=300 \mathrm{~s}$, a low-frequency field of amplitude 4 Vrms and frequency $100 \mathrm{~Hz}$ is applied. In doing so, the droplet flattens and rotates faster, always clockwise. Finally, the frequency is switched from $100 \mathrm{~Hz}$ to $10 \mathrm{kHz}$ at time $t=600 \mathrm{~s}$ while maintaining an amplitude of 4 Vrms. Under this condition, the droplet recovers its initial radius and rotates clockwise as if no field is applied. To better quantify this phenomenon, I systematically measure the angular velocity and the droplet deformation $D_{s}=\left(R_{1}-R_{2}\right) /\left(R_{1}+R_{2}\right)$ (with $R_{1}$ and $R_{2}$ being the principal radii of the droplet in the vertical and horizontal directions) as a function of frequency $f$ under constant voltage (4 Vrms) for droplets of radius $10 \mu \mathrm{m}$ in sample 3. The deformation is calculated from formula

$$
D_{s}=\frac{1-\left(R / R_{\infty}\right)^{3}}{1+\left(R / R_{\infty}\right)^{3}}
$$

where $R \equiv R_{2}$ is the apparent radius of the droplet measured on the photos and $R_{\infty}$ its radius measured at high frequency. This formula is obtained by noting that 1) the droplet volume $V$ does not change when the field is applied and 2) the droplet remains quasi-spherical [6] with the same radius as with no field at high frequency [31] (see Fig. 1). As a consequence $V=\frac{4}{3} \pi R_{\infty}^{3}=\frac{4}{3} \pi R_{1} R^{2}$, yielding $R_{1} / R=R_{\infty}^{3} / R^{3}$ from which Eq. (1) is deduced.

Fig. 2(a) shows that the angular velocity is constant at high and low frequency (with a tendency to decrease at very low frequency, in particular in the sample doped with $\mathrm{DTAB}^{1}$ ) and is almost 9 times larger at low frequency than at high frequency. In this experiment, the cutoff frequency clearly coincides with the charge relaxation frequency, the values of which in the two phases are indicated by the vertical arrows in the figure. Fig. 2(b) shows that this velocity change is also clearly correlated with a deformation of the droplets into a prolate shape $\left(D_{s}<0\right)$ in the lowfrequency regime.

To confirm that the cutoff frequency is well given by the charge relaxation frequency, I perform similar mea-

\footnotetext{
1 This is certainly due to the formation of Debye layers near
} the electrodes that screen the field, as evidenced by the dielectric measurements shown in the Appendix. 

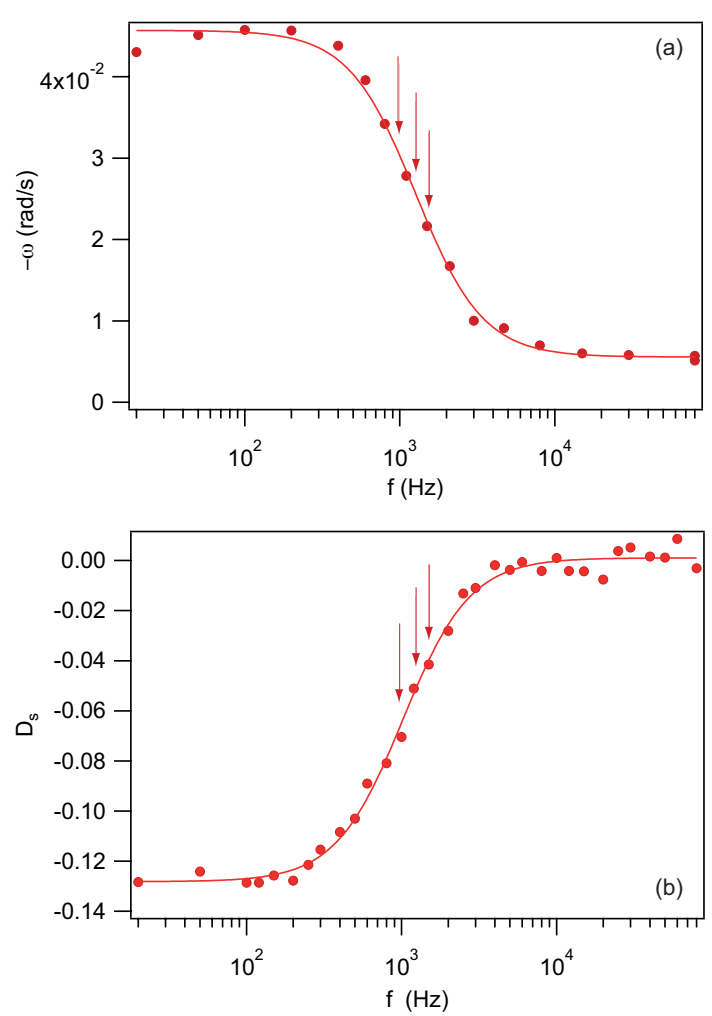

Fig. 2. Angular velocity $\omega\left(\right.$ a) and droplet deformation $D_{s}$

(b) as a function of frequency $f . \Delta T=2^{\circ} \mathrm{C}, V=4$ Vrms and $R=10 \mu \mathrm{m}$ and $d=21 \mu \mathrm{m}$. The three arrows from left to right indicate, respectively, the values of $f_{r}^{\|}, f_{r}^{\perp}$ and $f_{r}^{I}$-measured the same day for more confidence. Sample 3 was used for these measurements. The solid lines are fits with a law of the type $a+b /\left(1+c f^{2}\right)$ where $a, b$ and $c$ are the fit parameters.

surements with a sample doped with a small quantity of TBAB (sample 4). With this new sample, the charge relaxation frequency is almost two orders of magnitude larger than in the previous sample (sample 3). The new measurements are shown in Fig. 3 together with those of Fig. 2 by using the reduced frequency $f / \bar{f}_{r}$, where $\bar{f}_{r}=\left(f_{r}^{\perp}+f_{r}^{\|}+f_{r}^{I}\right) / 3$ is an average charge relaxation frequency. With this new variable the two velocity curves and the two deformation curves superimpose. This confirms
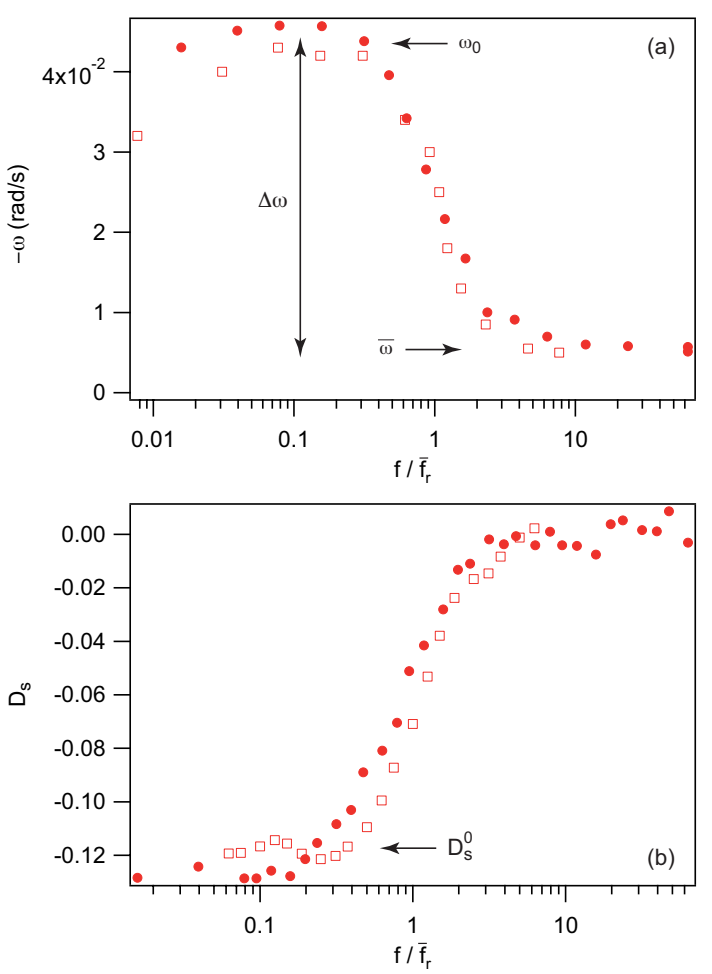

Fig. 3. Angular velocity $\omega$ (a) and droplet deformation $D_{s}$ (b) as a function of the reduced frequency $f / \bar{f}_{r}$ measured in two samples with very different charge relaxation frequencies. For sample 3 (filled circles), $\bar{f}_{r}=1290 \mathrm{~Hz}$ and for sample 4 (empty squares), $\bar{f}_{r}=64000 \mathrm{~Hz}$. In these two experiments, $\Delta T=2^{\circ} \mathrm{C}, V=4 \operatorname{Vrms}$ and $R=10 \mu \mathrm{m}$. These two samples have similar thicknesses $d \approx 21 \mu \mathrm{m}$.

that the droplets start to deform and rotate faster at the transition between the dielectric and conducting regimes when the frequency is decreased. Another important point is that the values of the velocity jump $\Delta \omega=\omega_{0}-\bar{\omega}$ and the deformation $D_{s}^{0}$ measured on the plateau in the conducting regime are independent of the concentration of ionic impurities that mainly fixes the cutoff frequency between the two regimes.

I also measure the effect of the field amplitude on the rotation velocity and the deformation of the droplets in 

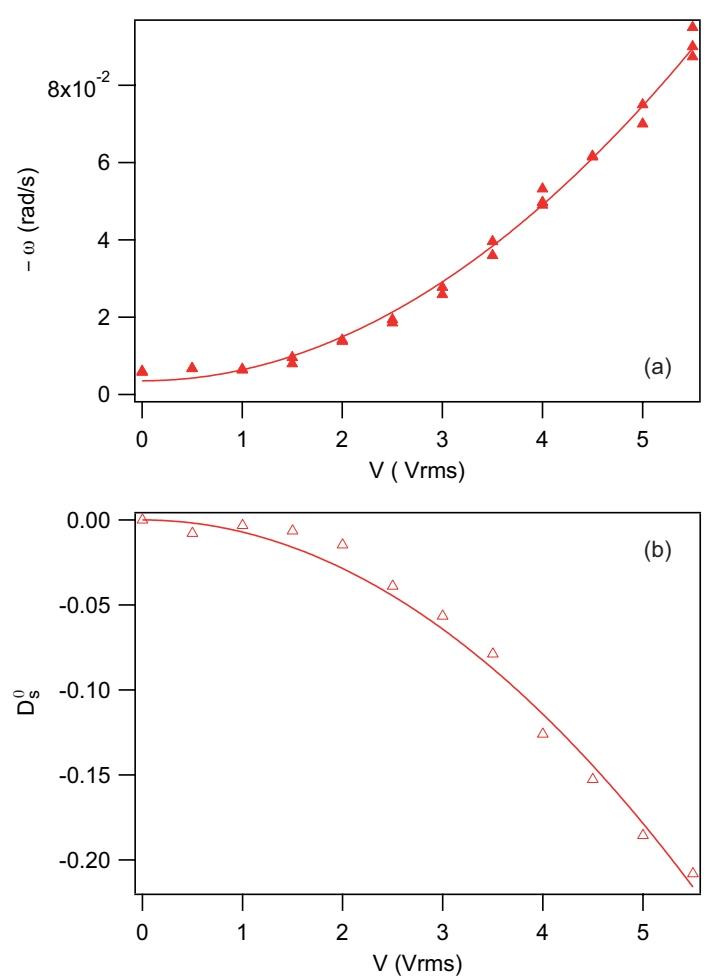

Fig. 4. Angular velocity $\omega$ (a) and droplet deformation $D_{s}^{0}(\mathrm{~b})$ of droplets of radius $R=10 \mu \mathrm{m}$ as a function of the applied voltage $V$ in the conducting regime at $f=100 \mathrm{~Hz}$. A small temperature gradient is applied $\left(\Delta T=2^{\circ} \mathrm{C}\right)$. Sample 3 was used for these measurements. The solid lines are the best fits with a law of the type $a+b V^{2}$, where $a$ and $b$ are the fit parameters.

the conducting regime. I find that these two quantities increase (in absolute value) quadratically with the applied field. This is shown in Fig. 4 for droplets of radius $R=$ $10 \mu \mathrm{m}$ subjected to a temperature gradient $\Delta T=2^{\circ} \mathrm{C}$.

\subsection{Role of the droplet radius}

In previous studies on the Lehmann effect, the rotation velocity of the banded droplets was found to decrease quadratically with their radius [11]. Does this dependence

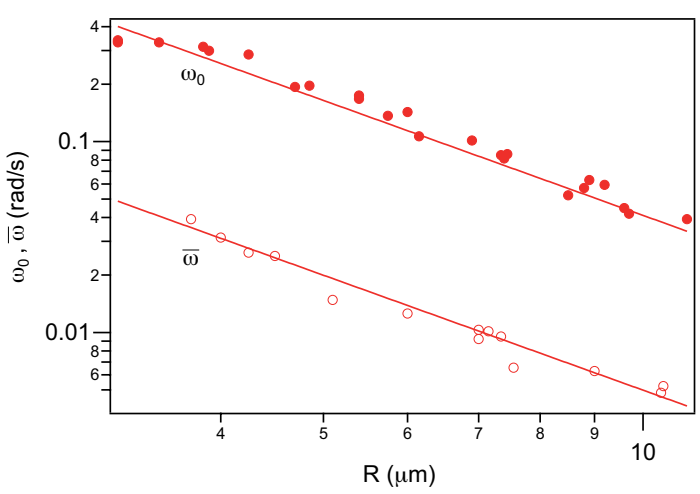

Fig. 5. Angular velocity as a function of the radius when $V=0$ $(\bar{\omega})$ and $V=4$ Vrms, $f=100 \mathrm{~Hz}\left(\omega_{0}\right)$. The solid lines are the best fits with a $1 / R^{2}$ law. Sample 1 with $\Delta T=2^{\circ} \mathrm{C}$.

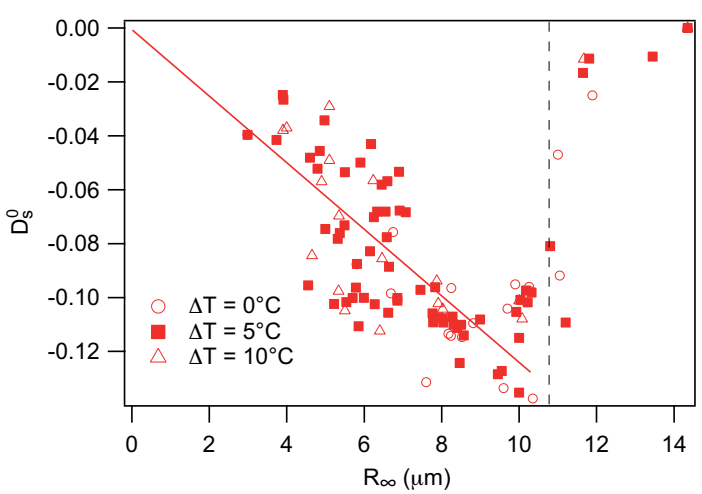

Fig. 6. Deformation $D_{s}^{0}$ as a function of the radius $R_{\infty}$ measured in sample 3 for three different values of $\Delta T$ at $V=4$ Vrms and $f=100 \mathrm{~Hz}$. The solid line is the best linear fit passing through the origin of the full data set for $R_{\infty}<10 \mu \mathrm{m}$. The vertical line indicates the value of the half sample thickness. The points on the right of this line correspond to confined droplets.

also apply for the velocity increase in the conducting regime when an electric field is applied ? To answer this question, I measure the rotation velocity as a function of the droplet radius in the absence $(\bar{\omega})$ and in the presence $\left(\omega_{0}\right)$ of an electric field in the conducting regime. Note that the radii reported here are the apparent radii of the droplets mea- 
sured on the photos. These measurements are performed in sample 1 at $V=4$ Vrms and $f=100 \mathrm{~Hz}$ in the conducting regime (see table 1). Typical velocity curves are reported in Fig. 5 when $\Delta T=2^{\circ} \mathrm{C}$, showing that $\omega_{0}$ and $\bar{\omega}$ both decreases as $1 / R^{2}$. A direct consequence is that the velocity jump $\Delta \omega=\omega_{0}-\bar{\omega}$ also decreases as $1 / R^{2}$. This dependence is observed in all samples at all temperature gradients.

I also measure the deformation $D_{s}^{0}$ on the plateau in the conducting regime as a function of the radius. Data obtained with sample 3 are shown in Fig. 6 for three different values of $\Delta T$. In practice, $D_{s}^{0}$ is measured at $100 \mathrm{~Hz}$ and the radius at $10 \mathrm{kHz}$ in the dielectric regime (noted $\left.R_{\infty}\right)$. This curve is noisy because of the difficulty to precisely measure the droplet radius. Nevertheless, the measurement shows that the smaller the drop, the smaller the deformation. This graph also shows that the deformation strongly decreases when the droplets become confined, with a diameter larger than the thickness, and touch the two electrodes.

\subsection{Role of the temperature gradient}

It is known that the velocity $\bar{\omega}$ is proportional to the temperature gradient [11]. Is it the same for the velocity jump $\Delta \omega$ ? To answer this issue, I systematically measure $\Delta \omega$ in samples 1-4 as a function of the temperature difference $\Delta T$ for droplets of radius $R=10 \mu \mathrm{m}$. The result is shown in

Fig. 7. This graph shows that $\Delta \omega$ is odd in $\Delta T$, meaning that the rotation velocity reverses when the temperature gradient is reversed. On the other hand, the absolute value

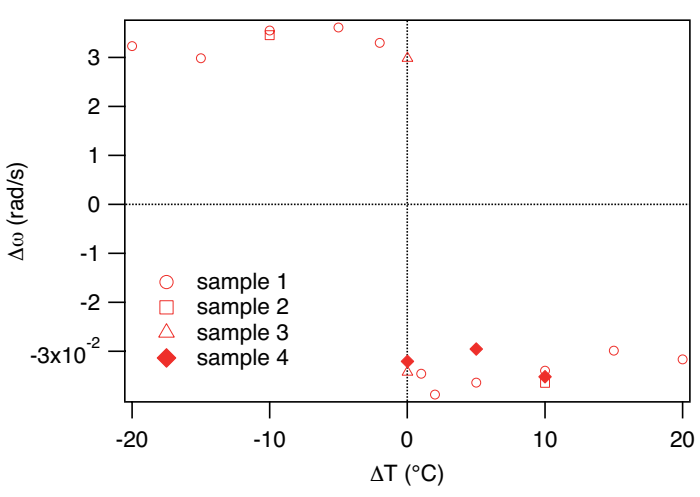

Fig. 7. Velocity jump $\Delta \omega$ as a function of the temperature difference $\Delta T$ measured in samples 1-4 of thickness $20 \mu \mathrm{m}$ at $V=4$ Vrms
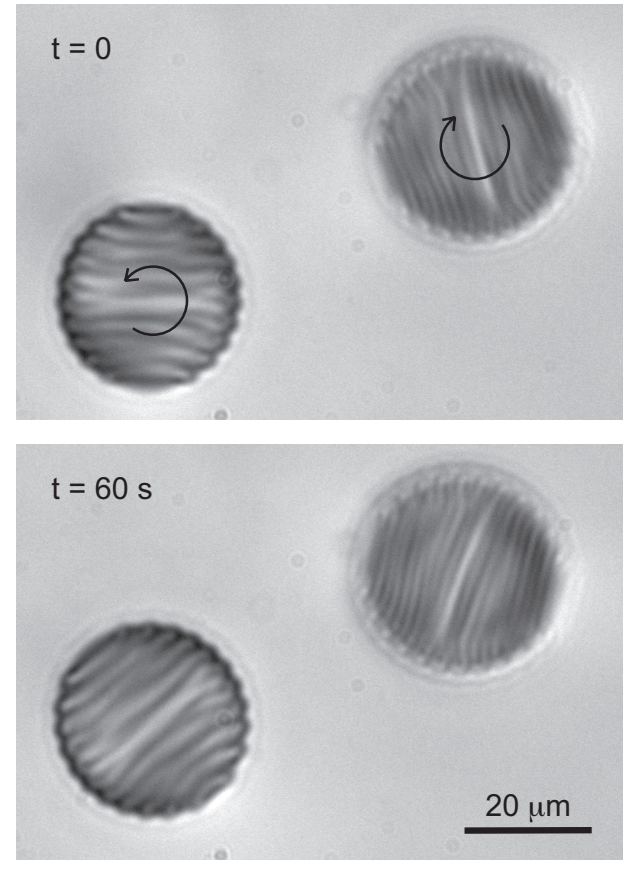

Fig. 8. Two droplets in sample 5 (of thickness $\sim 40 \mu \mathrm{m}$ ) rotating in opposite directions at $V=4 \mathrm{Vrms}$ and $f=100 \mathrm{~Hz}$ when the temperature is uniform $(\Delta T=0)$. The left droplet on focus under the microscope is attached to the top surface and the right one -out of focus- is attached to the bottom surface. 
of $\Delta \omega$ is independent of $\Delta T$ so that $\Delta \omega$ jumps from a negative to a positive value at $\Delta T=0$. Whence the question: what happens at $\Delta T=0$ ? To answer this question, I impose $\Delta T=0$ to sample 5 and observe the droplets under electric field in the conducting regime at $V=4 \mathrm{Vrms}$ and $f=100 \mathrm{~Hz}$. In doing so, I find that the droplets rotate in both directions depending on which plate they are in contact. More precisely, I observe that the droplets attached to the bottom plate rotate clockwise, while the others attached to the top surface rotate anti-clockwise. This is shown in Fig. 8 and the corresponding Video S2. In this figure and this video, the left droplet on which I focus is attached to the top plate and rotate anti-clockwise, while the other -which is out of focus- is attached to the bottom plate and rotates clockwise. The movie shows, in addition, that the two droplets attract, approach and finally coalesce when they contact each other. It is likely that this attractive force is hydrodynamic in nature and due to the convective rolls observed by Auernhammer et al. [14] in the isotropic liquid around the droplets. The fact that all the droplets rotate in the same direction when a temperature gradient is applied now explains easily. Indeed, imposing a temperature gradient forces the droplets to nucleate on the cold plate. As a consequence, all the droplets are in contact with the bottom plate when $\Delta T>0$ and rotate clockwise, while they are all in contact with the top plate and rotate anti-clockwise when $\Delta T<0$.

I also find that the deformation $D_{s}^{0}$ measured in the conducting regime is independent of the temperature gra-

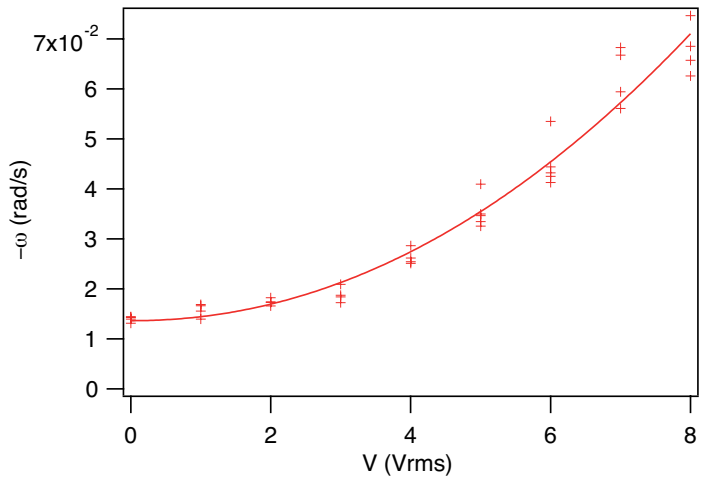

Fig. 9. Velocity $\omega$ measured with droplets of radius $R=10 \mu \mathrm{m}$ in sample 5 of thickness $\sim 40 \mu \mathrm{m}$ as a function of the applied voltage $V$ at $f=100 \mathrm{~Hz}$ and $\Delta T=5^{\circ} \mathrm{C}$. The best fit (solid line) with a power law of the type $a+b V^{\nu}$ gives $\nu=2.06 \pm 0.16$. This shows that $\Delta \omega$ increases quadratically with the electric field.

dient within the experimental errors. This is shown in Fig. 6 and in Fig. 10.

In conclusion, the velocity increase $\Delta \omega$ and the deformation $D_{s}^{0}$ are mainly due to the electric field, the temperature gradient only selecting the sense of rotation of the droplets by just imposing on which plate they are in contact.

\subsection{Role of the sample thickness}

It is already known that the velocity $\bar{\omega}$ of banded droplets is independent of the sample thickness [32]. I observe the same in the present experiments. However, the velocity increase $\Delta \omega$ should depend on the thickness if it is due to the flows observed by Auernhammer et al. [14]. Indeed, the thicker the sample, the stronger must be the flows, and the faster the droplets should rotate, if this interpretation is correct. 


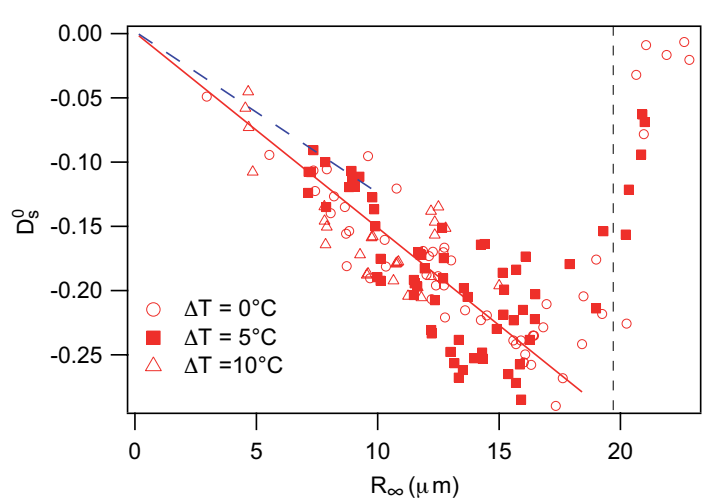

Fig. 10. Deformation $D_{s}^{0}$ as a function of the droplet radius $R$ measured for three different temperature gradients at $V=$ 8 Vrms and $f=100 \mathrm{~Hz}$ in sample 5 of thickness $\sim 40 \mu \mathrm{m}$. The solid red line is the best linear fit passing through the origin of the full data set for $R<18 \mu \mathrm{m}$. The vertical line indicates the value of the half sample thickness. The points on the right of this line correspond to confined droplets. The dashed blue line shows the same curve obtained with sample 3 of thickness $\sim 20 \mu \mathrm{m}$ under the same electric field ( $V=4 \mathrm{Vrms}$ and $f=100 \mathrm{~Hz}$ ) and similar temperature gradients.

To check this point, I prepare a sample twice thicker than the rest (sample 5 of thickness $40 \mu \mathrm{m}$, table 1 ) and I observe that $\Delta \omega$ still changes quadratically with the applied voltage (Fig. 9). I then measure $D_{s}^{0}$ as a function of $R_{\infty}$ and $\Delta \omega$ as a function of $\Delta T$ in the conducting regime, by taking $V=8$ Vrms and $f=100 \mathrm{~Hz}$ to keep the same electric field as in Figs. 6 and 7. The results are shown in Figs. 10 and 11. A comparison with the average data obtained from the $20 \mu \mathrm{m}$-thick samples (represented by the dashed lines in the two figures) shows that $D_{s}^{0}$ and $\Delta \omega$ increase in absolute value by a factor of 1.3 and 2 , respectively, when the thickness doubles at constant electric field. This confirms that the flows are certainly responsible

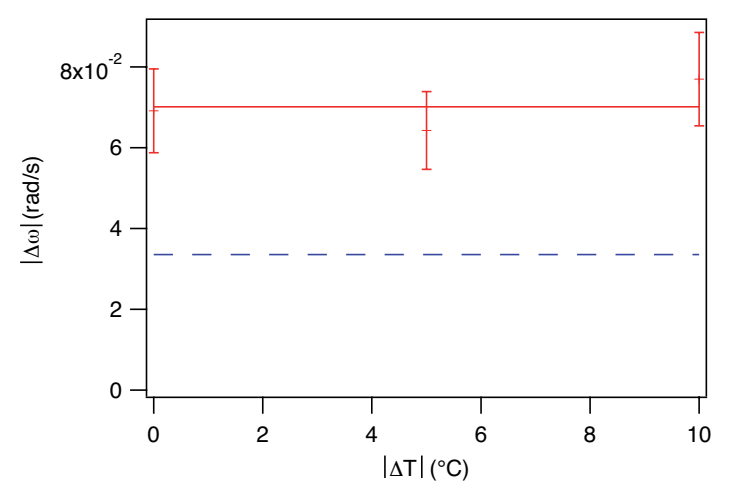

Fig. 11. Absolute value of the velocity jump $|\Delta \omega|$ measured with droplets of radius $R=10 \mu \mathrm{m}$ in sample 5 of thickness $\sim 40 \mu \mathrm{m}$ as a function of the absolute value of the temperature difference $|\Delta T|$ at $V=8 \mathrm{Vrms}$ and $f=100 \mathrm{~Hz}$. Within the experimental errors, $|\Delta \omega|$ is independent of $|\Delta T|$, of average value (solid red line) about twice larger than that measured in the $20 \mu$ m-thick samples (dashed blue line) at $V=4$ Vrms and $f=100 \mathrm{~Hz}$. Each error bar indicates the standard deviation over a dozen measurements.

for the increase of the rotation velocity of the droplets under the electric field in the conducting regime. The origin of these flows is discussed in the next section.

\section{Discussion}

The first remark concerns the symmetries. Indeed, it has been shown in Ref. [31] that the director field of a spherical banded droplet is invariant with respect to a $\pi$ rotation about a horizontal axis parallel or perpendicular to the bands. For this reason, such a droplet cannot rotate in average under an $\mathrm{AC}$ electric field if it is at equal distance from the electrodes. The only possibility to observe a rotation is to break this $C_{2}$ symmetry. This is indeed the case experimentally because the droplets are always in contact 
and, for this reason, slightly truncated, with one of the two plates limiting the sample.

The next question concerns the origin of the rotation. For the pure Lehmann effect (velocity $\bar{\omega})$, I refer to Ref. $[11,12]$ for a discussion on its origin. As for the velocity jump $\Delta \omega$ observed under electric field in the conducting regime, the present experiments suggest that it is due to the EHD flows. This is quite plausible since the velocity field couples to the director field to make the texture rotate with the angular velocity:

$$
\omega=\frac{\iiint_{\text {drop }}\left(\gamma_{2} \underline{A} \mathbf{n}+\gamma_{1}[(\mathbf{v} \cdot \nabla) \mathbf{n}-\mathbf{\Omega} \times \mathbf{n}]\right) \cdot \frac{\delta \mathbf{n}}{\delta \theta} \mathrm{d}^{3} \mathbf{r}}{\iiint_{\operatorname{drop}} \gamma_{1}\left(\frac{\delta \mathbf{n}}{\delta \theta}\right)^{2} \mathrm{~d}^{3} \mathbf{r}}
$$

In this formula, $\frac{\delta \mathbf{n}}{\delta \theta}=\frac{\partial \mathbf{n}}{\partial \theta}-\mathbf{e}_{z} \times \mathbf{n}$, by denoting by $\mathbf{e}_{z}$ the unit vector along the vertical $z$-axis and $\theta$ the polar angle in cylindrical coordinates $(r, \theta, z), \boldsymbol{\Omega}=\frac{1}{2} \nabla \times \mathbf{v}$ is the local rotation rate, $\underline{A}$ is the symmetric strain rate tensor of component $A_{i j}=\frac{1}{2}\left(\mathbf{v}_{i, j}+\mathbf{v}_{j, i}\right)$ and $\gamma_{1} \equiv \alpha_{3}-\alpha_{2}$ and $\gamma_{2} \equiv \alpha_{3}+\alpha_{2}$ are two viscosities of the cholesteric phase (with $\alpha_{2}$ and $\alpha_{3}$ two of the five Leslie viscosity coefficients $[1,2])$

This general formula is derivated in Ref. [13] and remains valid when an electric field is applied as can be easily checked by adding the electric energy to the elastic and surface anchoring energies in the derivation and by replacing the elastic molecular field $\mathbf{h}$ by $\mathbf{h}+\mathbf{h}^{E}$, where $\mathbf{h}^{E}=\varepsilon_{0}\left(\epsilon_{\|}-\varepsilon_{\perp}\right)(\mathbf{n} \times \mathbf{E}) \mathbf{E}$ is the so-called electric contribution to the molecular field. Note that the flexoelectric terms disappear under AC electric field because they are linear in $E$, and so they vanish on average over time.

In practice, the flows can have two origins, depending of the type of charges considered.

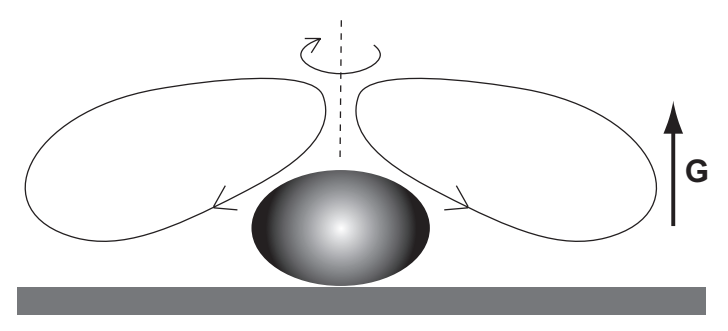

Fig. 12. Sketch of the flow field around a droplet observed by Auernhammer et al. by using tracer colloids (from Ref. [14]).

In the model of Tarasov, Krakhov and Kramer [21,22] the negative and positive charges separate in the bulk of the cholesteric phase in the conducting regime because of the anisotropy of the electrical conductivity. The sample remains electrically neutral in average. This is the CarrHelfrich effect, responsible for the convective instabilities in a nematic slab under electric field in the conducting regime $[1,2]$. Under the action of the electric field, these charges experience a Coulomb force, which generates flows in the cholesteric phase. These flows are responsible for the drift of cholesteric fingers under $\mathrm{AC}$ electric field in the conducting regime $[21,33]$ and could explain, at least in part, the velocity jump $\Delta \omega$ in the present experiments. This model is plausible as it predicts that $\mathbf{v}-$ and so $\Delta \omega$ according to Eq. (2)- must change as [21,22]

$$
\Delta \omega \propto \frac{E^{2}}{1+f^{2} / f_{r}^{2}}
$$

where $f_{r}$ is the typical charge relaxation frequency. Such a dependence on $f$ is indeed observed experimentally (see Fig. 2(a)), as well as the quadratic dependence on the electric field (see Fig. 4(a) and Fig. 9).

On the other hand, this model ignores the deformation of the droplets. The latter is due to the presence of charges 

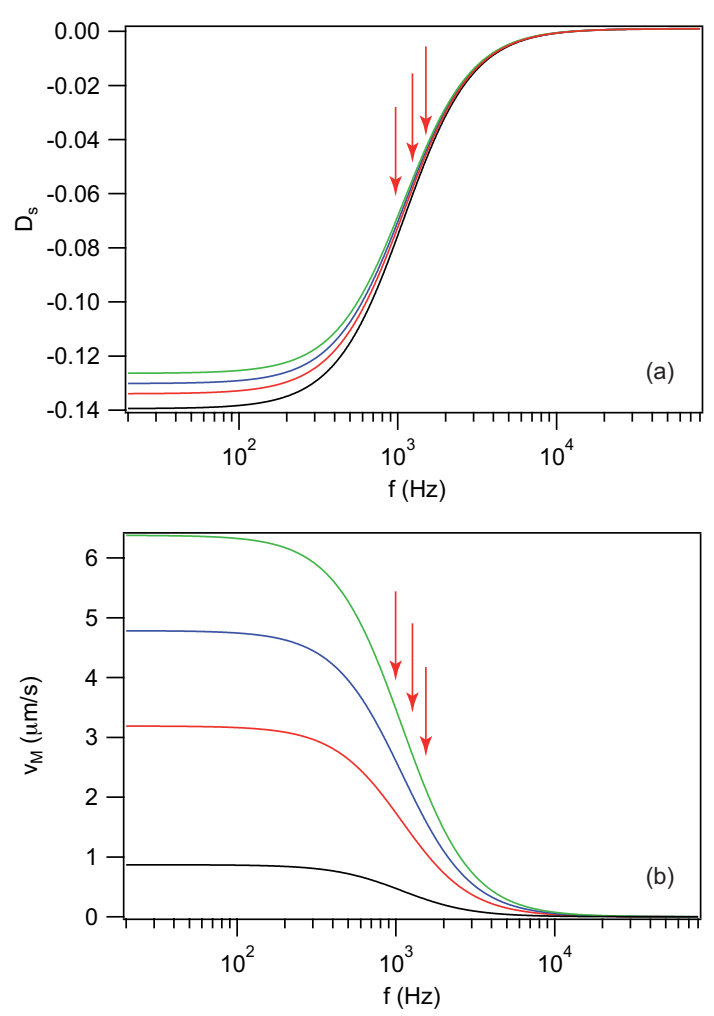

Fig. 13. Deformation $D_{s}$ (a) and maximum velocity $v_{M}$ (b) predicted by the Taylor-Melcher model by taking the values of the parameters corresponding to sample 3. In the graphs, the curves from top to bottom have been calculated by taking $M=0.5,1,2,10$. The three arrows from left to right indicate the values of $f_{r}^{\|}, f_{r}^{\perp}$ and $f_{r}^{I}$, respectively.

at their surface. Indeed, according to the Taylor-Melcher model, positive and negative charges also appear on the surface of the droplet, with a net charge remaining equal to 0 as in the bulk. The action of the field on these surface charges generate electric stresses across the cholestericisotropic interface, which not only leads to deformation of the droplets but also generates flows in and around the droplets, which should add to the flows created by the bulk charges.
The flow out of the droplets was observed experimentally by Auernhammer et al. [14] and is schematically sketched in Fig. 12 when the temperature gradient points upwards (droplet in contact with the bottom electrode). In this case, the direction of flow is the same as in the cholesteric emulsion experiments reported in Ref. [13] when the temperature gradient points upwards, which was shown to produce a rotation in the same sense as in the usual Lehmann effect. In practice, the confinement of the droplets between the two electrodes must alter the distribution of the electric field and the associated flows. This is shown in recent numerical simulations of confined two-dimensional isotropic droplets [34]. This effect could explain why the deformation $D_{s}^{0}$ and the velocity jump $\Delta \omega$ depend on the thickness in my experiments.

It is also important to note that the Taylor-Melcher model predicts the good order of magnitude for both $D_{s}$ and the velocity, of the order of a few $\mu \mathrm{m} / \mathrm{s}$ according to Auernhammer et al. [14]. To check this point, I plot the curve of the deformation $D_{s}$ as a function of frequency $f$ in Fig. 13(a) for a droplet of radius $R=10 \mu \mathrm{m}$ under an electric field $E=V / d=210^{5} \mathrm{~V} / \mathrm{m}$ (corresponding to $V=4$ Vrms when $d=20 \mu \mathrm{m}$ ), by using the formula [27]

$$
D_{s}=\frac{9 \varepsilon_{0} \varepsilon_{I}}{16 \gamma} \Phi_{s} R E^{2}
$$

valid for a non-confined droplet in a uniform electric field. In this formula, $\varepsilon_{0}$ is the permittivity of free space, $\gamma$ is the surface tension and $\Phi_{s}$ is given by 


$$
\Phi_{s}=1-\frac{T(11 M+14)+T^{2}[15(M+1)+S(19 M+16)]+15 T^{2}(1+M)(1+2 S) f^{2} / f_{r}^{I 2}}{5(1+M)\left[(2 T+1)^{2}+T^{2}(S+2)^{2} f^{2} / f_{r}^{I 2}\right]}
$$

where $S \equiv \varepsilon_{\text {chol }} / \varepsilon_{I}, T \equiv \sigma_{I} / \sigma_{\text {chol }}, M \equiv \mu_{\text {chol }} / \mu_{I}$ with $\mu$ the viscosity, and $f_{r}^{I}=\sigma_{I} / 2 \pi \varepsilon_{0} \varepsilon_{I}$. To plot this curve I assume that $\varepsilon_{\text {chol }} \approx\left(\varepsilon_{\|}+\varepsilon_{\perp}\right) / 2, \sigma_{\text {chol }} \approx\left(\sigma_{\|}+\sigma_{\perp}\right) / 2$ and I use the values of the parameters measured in sample 3, viz. $\varepsilon_{\|}=13, \varepsilon_{\perp}=7.5, \varepsilon_{I}=9.6$ and $\sigma_{\|}=6.3710^{-7} \mathrm{~S} / \mathrm{m}$, $\sigma_{\perp}=4.810^{-7} \mathrm{~S} / \mathrm{m}$ and $\sigma_{I}=6.1410^{-7} \mathrm{~S} / \mathrm{m}$. The surface tension is given by Faetti and Palleschi in $8 \mathrm{CB}$ : $\gamma=0.9510^{-5} \mathrm{~N} / \mathrm{m}[35]$. It turns out that the curve calculated with Eqs. (4) and (5) depends little on the value of the viscosity ratio $M$ and is very close to the experimental curve of Fig. 2(b) in spite of the fact that these formulas do not take into account the confinement of the droplet. This good agreement is certainly a coincidence. However, there is no doubt that this model leads to the correct order of magnitude and is pertinent, as already underlined by Auernhammer et al. [14].

This model also predicts that the maximum velocity at the surface of the droplet is frequency dependent, given by $[27]$

$$
v_{M}=\frac{9 \varepsilon_{0} \varepsilon_{I} E^{2} R}{20 \mu_{I}(1+M)} \frac{T(T S-1)}{(2 T+1)^{2}+T^{2}(S+2)^{2} f^{2} / f_{r}^{I}}
$$

The curve of $v_{M}$ as a function of $f$ is shown in Fig. 13(b). This curve shows the same behavior in frequency as the experimental curve for $\Delta \omega$, which is expected since $\Delta \omega$ is proportional to $v_{M}$ according to Eq. (2). The velocity is also of the order of a few $\mu \mathrm{m} / \mathrm{s}$, which is compatible with the observations of Auernhammer et al. [14]. As a consequence, the Taylor-Melcher model could also explain the rotation of the droplets under $\mathrm{AC}$ electric field in the conducting regime.

Another crucial point is that, in the two models, only the conductivity ratios enter into the calculations. If all the conductivities change in the same way with the concentration of ionic impurities, which is likely at small concentrations, this could also explain why the deformation $D_{s}^{0}$ and the velocity jump $\Delta \omega$ are experimentally independent of the concentration of ionic impurities (see Fig. 3).

For the moment, it is difficult to say which mechanism is the most important. However, there is no doubt that the surface charges are important to explain the deformation of the droplets. The confinement effects are also important to explain the thickness dependence of both the deformation and the rotation velocity of the droplets.

In the future it would important to solve numerically this problem by taking into account these two mechanisms. This is a very difficult task because of the complexity of the equations. A way to simplify the problem would be to calculate separately the velocity fields due to the Carr-Helfrich and Taylor-Melcher mechanisms. The contribution of each of them to the rotation velocity could then be calculated from the general equation (2) and compared. In these calculations, an additional simplification would be to consider that the director field is the same as at equilibrium, without flow. 
It would also be interesting to perform the experiments becomes sliding under UV [36]. It would also be interestwith other liquid crystals. Indeed, the Taylor-Melcher model ing to make the dielectric anisotropy of the LC vanish, predicts that depending on the values of the ratios of the for instance by mixing MBBA with a small amount of dielectric constants and conductivities, the electric field $8 \mathrm{CB}$ (or $5 \mathrm{CB}[37]$ ), to avoid texture changes under eleccan deform a droplet into a prolate or oblate ellipsoidal shape. With $8 \mathrm{CB}$, an oblate shape is observed and the flows make the texture rotate in the same direction as in the Lehmann effect. As a consequence the two effects add. By contrast, a prolate shape should produce an opposite effect since the velocity changes sign. In that case, the electric field should make the texture rotate in the oppotric field. Finally, it would be interesting to visualize the flows by doping the LC with colloids, as in the experiments of Auernhammer et al. [14]. Such experiments are planed for the future.

\section{Acknowledgements}

P. O. warmly thanks G. Poy, A. Dequidt and J. IgnésMullol for their critical reading of the manuscript. site direction of the Lehmann effect. It turns out that I observe such a phenomenon with a sample of MBBA (pmethoxybenzylidene-p'-butylaniline) doped with $1.5 \mathrm{wt} \%$ R811. In this mixture, the droplets deform very little but slow down and start to rotate in the opposite direction when an increasing electric field is applied. Unfortunately, the experiments with MBBA are complicated because the texture of the droplets change under electric field, the helix tending to align along the field because of the negative dielectric anisotropy of the LC. For this reason, it is difficult to determine whether this effect is due to a texture change or to a reversal of the flows. In addition, the polymercaptan dissolves in MBBA, so that the transition temperature of the samples constantly decreases. For this reason, the droplets melt during the measurements, which become very imprecise. A solution to solve this issue could be to change the surface treatment, for instance by using the azobenzene polymer poly(ethylene oxide-b-11-[4(4-butylphenylazo)phenoxy]-undecyl methacrylate which

\section{A Measurement of the charge relaxation}

\section{frequency}

In the leaky dielectric model, the liquid crystal is treated as a dielectric medium with an Ohmic resistance. Let $\varepsilon$ be the dielectric constant and $\sigma$ the conductivity of the LC. The charge relaxation frequency

$$
f_{r}=\frac{\sigma}{2 \pi \varepsilon_{0} \varepsilon}
$$

defines the limit between the conducting regime $\left(f<f_{r}\right)$ and the dielectric regime $\left(f>f_{f}\right)$. It can be calculated if the resistance $R_{p}=\frac{d}{\sigma S}$ and the capacitance $C_{p}=\varepsilon_{0} \varepsilon \frac{S}{d}=$ $\varepsilon C_{0}$ of the sample are known (Fig. A.1), by denoting by $d$ the sample thickness, $S$ its surface area and $C_{0}$ its capacitance without the LC.

To obtain $C_{p}$ and $R_{p}$, the complex impedance $R+j X$ of the sample is measured as a function of frequency $f$ and 


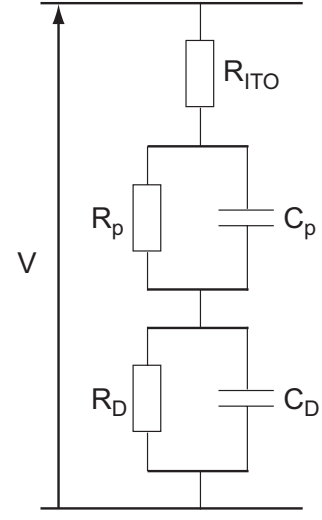

Fig. A.1. Electric representation of the sample. The LC layer is modeled by a resistance $R_{p}$ in parallel with a capacitance $C_{p}$. The resistance $R_{D}$ and the capacitance $C_{D}$ are introduced to simulate the effect of the Debye layers that form at low frequency close to the electrodes [40]. The two ITO layers are replaced by the resistance $R_{I T O}$.

is then fitted with formulas

$$
\begin{aligned}
& R=R_{I T O}+\frac{R_{p}}{1+4 \pi^{2} R_{p}^{2} C_{p}^{2} f^{2}}+\frac{R_{D}}{\left.1+4 \pi^{2} R_{D}^{2} C_{D}^{2} f^{2} \mathrm{~A} .2\right)} \\
& X=-\frac{2 \pi R_{p}^{2} C_{p} f}{1+4 \pi^{2} R_{p}^{2} C_{p}^{2} f^{2}}-\frac{2 \pi R_{D}^{2} C_{p} f}{1+4 \pi^{2} R_{D}^{2} C_{D}^{2} f^{2}}
\end{aligned}
$$

Two examples of global fit performed with IGOR pro (Version 6.37. WaveMetrics, Inc.) are shown in Fig. A.2. Their good quality is a clear indication that the leaky dielectric model is well suited to describe the electric behavior of the $\mathrm{LC}$ when it is pure (Fig. A.2a) or doped with TBAB (Fig. A.2b). From each fit, the values of $R_{p}$ and $C_{p}$ are deduced from which $f_{r}=1 /\left(2 \pi R_{p} C_{p}\right)$ is calculated as well as the dielectric constant $\varepsilon=C_{p} / C_{0}$ and the conductivity $\sigma=2 \pi \varepsilon f_{r}$

In practice, these measurements are performed for each sample in the isotropic liquid just above the liquidus temperature, and in the cholesteric phase just below the solidus
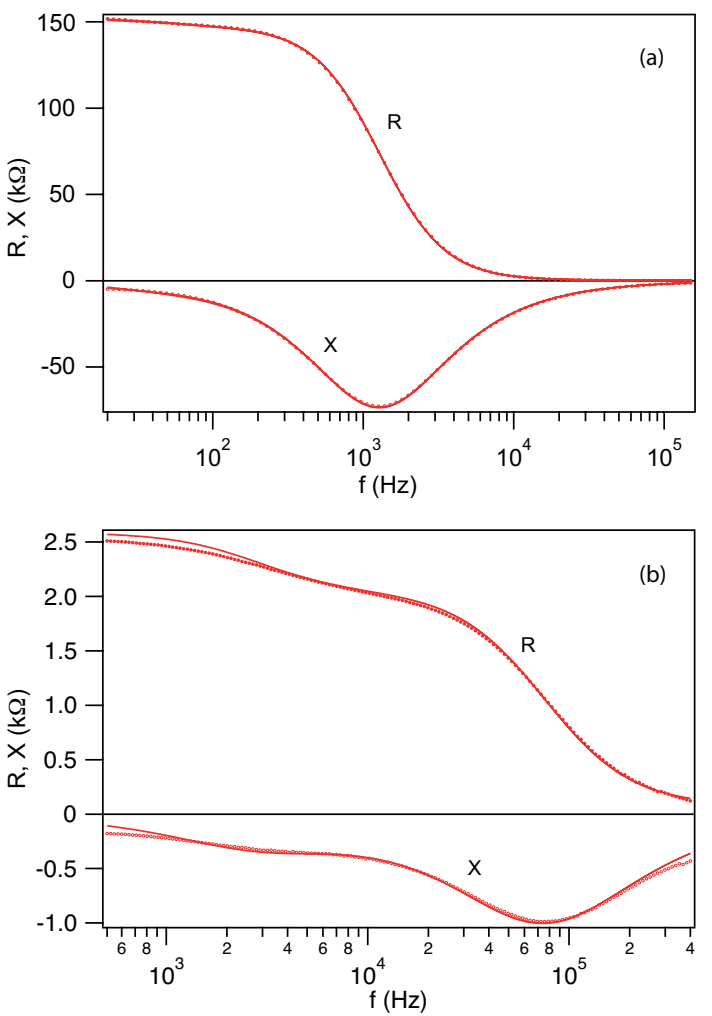

Fig. A.2. (a) Complex impedance of sample 3 measured in the planar orientation just below the solidus temperature and their fits with Eqs. (A.2) and (A.3) using a global fit procedure. From the fit parameters $R_{I T O}=100 \Omega, R_{p}=147 \mathrm{k} \Omega, C_{p}=843 \mathrm{pF}$, $C_{D}=726 \mathrm{nF}, R_{D}=4.4 \mathrm{k} \Omega$, I calculate $f_{r}^{\perp}=1280 \mathrm{~Hz}$ and $\varepsilon_{\perp}=7.6$, knowing that $C_{0}=111 \mathrm{pF}$; (b) Complex impedance of sample 4 (doped with TBAB) measured in the isotropic liquid just above the transition and their fits with Eqs. (A.2) and (A.3) using a global fit procedure. From the fit parameters $R_{I T O}=80 \Omega, R_{p}=1.96 \mathrm{k} \Omega, C_{p}=1074 \mathrm{pF}, C_{D}=102 \mathrm{nF}$, $R_{D}=0.5 \mathrm{k} \Omega$, I calculate $f_{r}^{I}=75600 \mathrm{~Hz}$ and $\varepsilon_{I}=9.6$, knowing that $C_{0}=112 \mathrm{pF}$.

temperature. In the latter case, measurements are performed at low voltage (0.5 Vrms) when the cholesteric phase is in planar orientation (director perpendicular to the field) and at large voltage (20 Vrms) when the cholesteric 
phase is completely unwound and in homeotropic orientation (director parallel to the field). From these measurements, I obtain the values of $f_{r}, \varepsilon$ and $\sigma$ in the isotropic liquid $\left(f_{r}^{I}, \varepsilon^{I}\right.$ and $\left.\sigma^{I}\right)$ and in the cholesteric phase under $\operatorname{planar}\left(f_{r}^{\perp}, \varepsilon^{\perp}\right.$ and $\left.\sigma^{\perp}\right)$ and homeotropic $\left(f_{r}^{\|}, \varepsilon^{\|}\right.$and $\left.\sigma^{\|}\right)$ orientations. For $8 \mathrm{CB}$, I find that in all samples $\varepsilon_{\perp} \approx 7.5$, $\varepsilon_{\|} \approx 13$ and $\varepsilon_{I} \approx 9.6$. These values compare well with the ones given in the literature $[38,39]$. The values of $f_{r}$ for the different samples are given in Table 1. Contrary to the dielectric constants, they depend on the purity of the sample and its content in ionic impurities.

\section{References}

1. P.G. de Gennes The physics of liquid crystals (Clarendon press, Oxford, 1974).

2. P. Oswald, P. Pieranski Nematic and Cholesteric Liquid Crystals: Concepts and Physical Properties Illustrated by Experiments (Taylor \& Francis, CRC press, Boca Raton, 2005).

3. O. Lehmann, Ann. Phys. (Leipzig) 307, 649 (1900).

4. P. Oswald, A. Dequidt, Phys. Rev. Lett. 100, 217802 (2008)

5. J. Yoshioka, F. Ito, Y. Suzuki, Y Tabe, Soft Matter 10, 5869 (2014).

6. T. Yamamoto, M. Kuroda, M. Sano, Europhys. Lett. 109, 46001 (2015).

7. J. Yoshioka, F. Araoka, Nat. Commun. 9, 432 (2018).

8. F.M. Leslie, Proc. R. Soc. A 307, 359 (1968).

9. P. Oswald, Eur. Phys. J. E 35, 10 (2012).

10. P. Oswald, Europhys. Lett. 108, 36001 (2014). Erratum: 108, 59901 (2014).
11. P. Oswald, A. Dequidt, G. Poy, Liq. Cryst. Rev. 7, 142 (2019)

12. P. Oswald, G. Poy, Phys. Rev. E 98, 032704 (2018).

13. P. Oswald, J. Ignès-Mullol, A. Dequidt, Soft Matter 15, 2591 (2019).

14. G.K. Auernhammer, J. Zhao, B. Ullrich, D. Vollmer, Eur. Phys. J. E. 30, 387 (2009).

15. J. Yoshioka, K. Fukao, Phys. Rev. E 99, 022702 (2019).

16. N.V. Madhusudana, R. Pratibha, Mol. Cryst. Liq. Cryst. 5, $43(1987)$.

17. N.V. Madhusudana, R. Pratibha, Liq. Cryst. 5, 1827 $(1989)$

18. Yu.I. Timirov, O.S. Tarasov, O.A. Skaldin, Tech. Phys. Lett. 33, 209 (2007).

19. Yu.I. Timirov, O.A. Skaldin, E.R. Basyrova, Tech. Phys. Lett. 41, 336 (2015).

20. A. Dequidt, P. Oswald, Eur. Phys. J. E. 24, 157 (2007).

21. O.S. Tarasov, A.P. Krekhov, L. Kramer, Phys. Rev. E 68, 031708 (2003).

22. O.S. Tarasov, A.P. Krekhov, L. Kramer, Rotation of cholesteric drops under $d c$ electric field, preprint (2003). This paper was never published after the death of L. Kramer.

23. O. Tarasov, Stuctural Transitions and Dynamics of Liquid Crystals under Flows and Electric Fields, PhD Thesis, Bayreuth University, 2003.

24. O.A. Skaldin, O.S. Tarasov, Yu.I. Timirov, E.R. Basyrova, JETP 126, 255 (2018).

25. G.I. Taylor, Proc. R. Soc. Lond. A 291, 159 (1966).

26. J.R. Melcher, G.I. Taylor, Ann. Rev. Fluid Mech. 1, 111 (1969).

27. S. Torza, R.G. Cox, S.G. Mason, Phil. Trans. R. Soc. Lond. 269, 259 (1971). 
28. P. Oswald, A. Dequidt, A. Żywociński, Phys. Rev. E 77, 35. S. Faetti, V. Palleschi, Phys. Rev. A 30, 3241 (1984). $061703(2008)$.

29. P. Oswald, Europhys. Lett. 100, 26001 (2012).

30. P. Oswald, G. poy, F. Vittoz, V. Popa-Nita, Liq. Cryst. 40, 734 (2013).

36. S. Bono, Y.Maruyamaa, K. Nishiyamaa, Y. Tabe, Mol. Cryst. Liq. Cryst. 683, 39 (2019).

31. G. Poy, F. Bunel, P. Oswald, Phys. Rev. E 96, 012705 (2017).

32. P. Oswald, G. Poy, Phys. Rev. E 91, 032502 (2015).

33. J. Baudry, S. Pirkl, P. Oswald, Phys. Rev. E 60, 2990 (1999).

34. S. Santra, S. Mandal, S. Chakraborty, Phys. Fluids 30, 062003 (2018). (1976).

37. A.G. Petrov, G. Durand, Liq. Cryst. 17, 543 (1994).

38. B.R. Ratna, R. Shashidhar, Mol. Cryst. Liq. Cryst. 6, 278

39. J. Thoen, G. Menu, Mol. Cryst. Liq. Cryst. 97, 163 (1983).

40. L.M. Blinov Electro-optical and magneto-optical properties of liquid crystals (John Wiley \& Sons, Hoboken, 1983). 